\title{
Monitoring in language perception: Electrophysiological and hemodynamic responses to spelling violations
}

\author{
Nan van de Meerendonk ${ }^{a}, *$, Peter Indefrey ${ }^{b}$, Dorothee J. Chwilla ${ }^{a}$, Herman H.J. Kolk ${ }^{a}$ \\ a Radboud University Nijmegen, Donders Institute for Brain, Cognition and Behaviour, The Netherlands \\ ${ }^{\mathrm{b}}$ Heinrich-Heine-Universität Düsseldorf, Institute for Language and Information, Germany
}

\section{A R T I C L E I N F O}

Article history:

Received 12 March 2010

Revised 4 October 2010

Accepted 7 October 2010

Available online 16 October 2010

\section{Keywords:}

Cognitive control

Conflict

Left inferior frontal gyrus

P600

Reprocessing

\begin{abstract}
A B S T R A C T
The monitoring theory of language perception proposes that competing representations that are caused by strong expectancy violations can trigger a conflict which elicits reprocessing of the input to check for possible processing errors. This monitoring process is thought to be reflected by the P600 component in the EEG. The present study further investigated this monitoring process by comparing syntactic and spelling violations in an EEG and an fMRI experiment. To assess the effect of conflict strength, misspellings were embedded in sentences that were weakly or strongly predictive of a critical word. In support of the monitoring theory, syntactic and spelling violations elicited similarly distributed P600 effects. Furthermore, the P600 effect was larger to misspellings in the strongly compared to the weakly predictive sentences. The fMRI results showed that both syntactic and spelling violations increased activation in the left inferior frontal gyrus (IIFG), while only the misspellings activated additional areas. Conflict strength did not affect the hemodynamic response to spelling violations. These results extend the idea that the IIFG is involved in implementing cognitive control in the presence of representational conflicts in general to the processing of errors in language perception.
\end{abstract}

(C) 2010 Elsevier Inc. All rights reserved.

\section{General introduction}

"Mistakes are a fact of life. It is the response to error that counts" (Nikki Giovanni). This quote nicely illustrates that we are bound to make mistakes but that we can also use the thereby obtained information to adjust our behaviour. To learn from our mistakes, however, we must be able to detect them. The detection of errors and the adjustments in behaviour require cognitive control. Cognitive control processes manage and guide other cognitive processes according to internal goals. One aspect of cognitive control is monitoring, which deals with the detection and repair of errors. Errors occur when there is a mismatch between what we intend or expect and what we do or observe. When such a mismatch is detected, compensatory adjustments are triggered in the processing pathways that are involved, leading to a repair.

Mistakes, of course, occur in various domains, and here we are interested in the language domain. In this field, the main focus has been on error monitoring in language production. For language production, researchers have been interested in speech errors and their repairs (for a review see Levelt, 1983). However, in language perception error monitoring takes place as well. For example, sometimes we mishear what another person is saying and ask for a

\footnotetext{
* Corresponding author. Donders Institute for Brain, Cognition and Behaviour, Radboud University Nijmegen, P.O. Box 9104, 6500 HE Nijmegen, The Netherlands. Fax: + 31243616066

E-mail address: N.vandeMeerendonk@donders.ru.nl (N. van de Meerendonk).
}

clarification: "Did I understand you correctly...?". Another type of perceptual language errors are mistakes that are made while reading a text. Kaufman and Obler (1995) call these 'slips of the eye', and showed that normal adult readers make various kinds of these errors. For instance, sometimes words are inserted, omitted or exchanged, the parsing of a word or sentence can be erroneous (e.g., reading posts as a noun instead of as a verb) or the wrong interpretation of a homonym can be selected (e.g., interpreting rock in rock art to mean a stone while based on the context it meant a form of music). Of course, there are also mistakes that are actually present in the text itself. For example, while proofreading your article you may encounter some spelling errors. Most of the time we will detect the errors because the interpretation will not fit into the wider context. Based upon this context, expectations are generated and when these are not met, a conflict arises between what was expected and what is observed. This results in a state of indecision: "Did I read that correctly?".

A number of electroencephalography (EEG) studies of language comprehension have linked this process of monitoring for such a representational conflict and triggering of compensatory adjustments to the P600 component. The P600 is a central-posterior distributed, positive-going event-related potential (ERP) component that starts around $500 \mathrm{~ms}$ and generally extends up to at least $800 \mathrm{~ms}$. An increase in P600 amplitude has been reported to various syntactic violations (e.g., Friederici et al., 1993; Hagoort et al., 1993; Münte et al., 1998), locally ambiguous garden-path sentences (Osterhout and Holcomb, 1992) and relatively complex sentences compared to simpler sentences (Kaan et al., 2000). These elicitations of a so called 
P600 effect to syntactic difficulties led to the proposal that the P600 reflects some form of syntactic processing (e.g., Friederici et al., 1996; Kaan et al., 2000). However, based on an increase in P600 amplitude to orthographic violations as well as to syntactic violations, Münte et al. (1998) proposed that the P600 does not just reflect syntactic processing but a more general reanalysis. A P600 to spelling violations has recently been replicated in our laboratory (see Vissers et al., 2006).

The hypothesis that the P600 effect reflects a more general reanalysis has been strengthened by a number of recent studies (for reviews on these studies see Kolk and Chwilla, 2007; Kuperberg, 2007; Van de Meerendonk et al., 2009), demonstrating P600 effects to different kinds of semantic anomalies (e.g., Ganushchak and Schiller, 2010; Hoeks et al., 2004; Kim and Osterhout, 2005; Kolk et al., 2003; Kuperberg et al., 2006, 2007, 2003b; Van de Meerendonk et al., 2010; Van Herten et al., 2006, 2005). Furthermore, P600 effects were found to picture-sentence mismatches in which the sentences violated the semantics of a previously shown picture (Vissers et al., 2008). The P600 effects to semantic anomalies were unexpected, as it was the general assumption that semantic anomalies affected the N400, a negative ERP component that peaks around $400 \mathrm{~ms}$ after critical word onset (e.g., Kutas and Hillyard, 1984).

According to the monitoring theory of language perception (for a review see Van de Meerendonk et al., 2009), common to all these studies is the assumption that there was a strong expectation for a certain linguistic element which was not met. This resulted in the coactivation of competing representations, i.e., the expected and the observed element. It is proposed that such competing representations trigger a conflict which functions as a bottom-up signal to elicit reprocessing of the input to check for possible processing errors, which is reflected by the P600 effect.

The previously-mentioned EEG studies that investigated monitoring processes in language perception manipulated two different factors: the type of material that elicited a conflict and the conflict strength. As said, various semantic anomalies, picture-sentence mismatches and spelling violations have been studied and it was found that representational conflicts of various types elicit a P600 effect. Some of these studies also directly compared the obtained P600 effects to the P600 effects elicited by morphosyntactic violations (e.g., subject-verb agreement violations) and found similar scalp distributions (e.g., Kuperberg et al., 2006; Van Herten et al., 2005). The second factor, conflict strength, has been manipulated in some studies based on the idea that for reasons of efficiency only representational conflicts that are sufficiently strong (i.e., pass a certain 'threshold') should trigger reprocessing of the input. For example, Van de Meerendonk et al. (2010) induced differences in conflict strength by varying plausibility. In the sentence context the critical words could either be plausible, mildly implausible, or deeply implausible. For instance:

(1) Lichaamsdelen zoals een arm, nek en teen/haar/telescoop ... (plausible/mildly implausible/deeply implausible)

Parts of the body like an arm, neck and toe/hair/telescope ... (literal translation)

Although both the mildly and deeply implausible condition violated the expectation based on the sentence context, only the deeply implausible condition was predicted to create a representational conflict of sufficient size. Therefore, only for this condition reprocessing and a P600 effect should be triggered. By contrast, it was hypothesized that the mildly implausible condition would lead to integration difficulties that could be resolved without reprocessing the input. The results confirmed these predictions. Compared to the plausible condition, the mildly implausible condition elicited an N400 effect (i.e., a more negative N400 amplitude). The deeply implausible condition elicited an N400 effect followed by a P600 effect. Vissers etal. (2006) manipulated conflict strength by varying cloze probabil- ity. The cloze probability of a certain word is the percentage of individuals that complete a sentence with that particular word. Vissers et al. (2006) found a P600 effect to misspelled words when the expected critical word in its correct spelling had a high cloze probability. Misspellings of critical words with a low cloze probability did not elicit a P600 effect.

In a recent study by Ganushchak and Schiller (2010) an effect of conflict strength was indicated as well. In this study participants saw a visual network of coloured objects while listening to a path description through this network. The path description could contain semantic (wrong colour name) or syntactic violations (determiner gender agreement error). An important difference between both types of violations was that colour errors could be detected based on both auditory information and visual information in the network, while determiner gender agreement errors could be detected based on auditory information only. Thus, participants could expect to hear a certain colour based on the visual network, but the retrieval of the concept of the word and its gender was needed to form an expectation for a certain determiner. Because the expectations formed for a certain colour were higher than for a certain determiner, the authors proposed that the semantic violations created a stronger conflict than the syntactic violations. In agreement with this proposal, the results showed that the semantic violations were more often detected than the syntactic ones. Furthermore, the condition with the stronger conflict, the semantic violations, elicited larger P600 amplitudes compared to correct trials, while syntactic violations did not.

Representational conflicts have also been investigated in functional magnetic resonance imaging (fMRI) studies. A brain area that is implicated in several of these studies is the posterior part of the left inferior frontal gyrus (Brodmann's area (BA) 44 and 45, also referred to as Broca's area, henceforth indicated as IIFG). The IIFG is part of the prefrontal cortex (PFC), a brain area that is thought to be important for cognitive control. As explained previously, monitoring is an aspect of cognitive control that is important when different responses or representations compete to be selected. The PFC, including IIFG, is thought to be involved in this process through resolving the competition between representations by biasing neural activity in the appropriate pathways (e.g., Miller and Cohen, 2001).

A task involving representational conflict that has been studied extensively in relation to cognitive control is the Stroop task (Stroop, 1935). In this task participants have to name the ink colour of a word and ignore the printed word. In the incongruent condition the ink colour to be named differs from the colour word that is printed (e.g., red printed in blue ink), thereby creating a conflict. Cognitive control is needed to shift attention away from the stronger task-irrelevant representation (the printed word) and direct attention towards the weaker task-relevant stimulus information (the ink colour). Incongruent Stroop trials have been found to elicit activation in the IIFG (e.g., see Nee et al., 2007; Novick et al., 2005).

In the language domain, a well-known example in which various representations are co-activated are so called garden-path sentences, which are sentences that are locally ambiguous and have a preferred parse, like:

\section{(2) The broker persuaded to sell the stock ...}

Garden-path sentences have been observed to elicit a P600 effect in the EEG (e.g., Osterhout and Holcomb, 1992, see previous discussion) and in fMRI they show increased activation in the IIFG (e.g., Mason et al., 2003).

Novick et al. (2005) proposed a unifying account for IIFG findings in the cognitive control literature and the psycholinguistic literature (see also Thompson-Schill et al., 2005). They proposed that the area is involved in general conflict resolution. To prevent misinterpretations, the IIFG is thought to implement cognitive control when there is a conflict between competing representations by biasing the activation patterns associated with the competing representations. The authors 
therefore suggest that, analogous to conflict resolution in the Stroop task, recovering from garden-path sentences (i.e., selecting the relevant parse) involves the IIFG to exert cognitive control and shift attention towards the relevant parse. To achieve reanalysis, the initially preferred parse needs to be suppressed and then all the evidence needs to be taken into account to recover the correct parse. In a recent study, January et al. (2009) directly compared the activation in the IIFG during representational conflict in the Stroop task and syntactically ambiguous sentences within subjects. They found that both types of conflict increased activation in the IIFG to a similar extent, and concluded that this is based on a shared conflict resolution mechanism.

Recent fMRI studies of language perception have investigated representational conflicts elicited by semantic anomalies in unambiguous sentences that had previously been shown to elicit P600 effects in the EEG. Kuperberg et al. (2008) tested 'animacy semanticthematic violations' (see also Kuperberg et al., 2006). The following is an example:

\section{(3) Every morning at breakfast the eggs would eat ...}

In this sentence, the syntactic parse indicates that the noun phrase (NP) the eggs is the agent of the sentence and therefore eat would be a syntactically correct verb form. However, the semantic characteristics of the NP in combination with the verb eat create the expectation that the NP is the theme of the action and should be accompanied by a passive verb form. The fMRI study revealed that both these animacy semantic-thematic violations and morphosyntactic (subject-verb agreement) violations elicited activation in a widespread network, which included part of the IIFG (BA 44). Another fMRI study of language perception (Ye and Zhou, 2009a) also investigated semantic anomalies in unambiguous sentences, in particular 'semantic reversal anomalies'. Semantic reversal anomalies are sentences in which the representation based on world knowledge and the representation based on the syntactic parse compete (e.g., Kolk et al., 2003; Van Herten et al., 2005; Ye and Zhou, 2008). For example:

(4) The thief kept the policeman in the police station (paraphrase of Chinese example)

Ye and Zhou (2009a) showed that a network of dorsal medial superior frontal gyrus, left inferior parietal lobule (IIPL) and IIFG was activated for these semantic reversal anomalies. In addition, they provided further support for a general conflict resolution mechanism, since the same network was found to be active for the incongruent conditions of Stroop and Flanker ${ }^{1}$ tasks (see also Ye and Zhou, 2009b).

In the present study we further explored the representational conflicts underlying the detection and reprocessing of errors in language perception. To this end, we conducted an EEG and fMRI experiment on syntactic and spelling violations. Our purpose was twofold. First, conflicts induced by syntactic and spelling violations have been found to elicit positivities in the EEG with very similar scalp distributions. Therefore, our question was whether we would also find co-localization of activity elicited by syntactic and spelling conflicts in the fMRI, possibly in the IIFG, supporting the assumption of a role of this area in general conflict resolution. Secondly, we were interested whether the effect of a conflict strength manipulation on the processing of spelling violations would also modulate the hemodynamic response in the fMRI. Whether or not the hemodynamic response of a certain region is affected by the strength of a representational conflict could be informative with respect to its role in the processing of a conflict and/or subsequent reanalysis processes.

\footnotetext{
1 In the Flanker task (Eriksen and Eriksen, 1974) people have to respond to, for example, a central arrow that is surrounded by other arrows. In the incongruent condition the central arrow is surrounded by arrows that point in a different direction (e.g., $\rightarrow \rightarrow \leftarrow \rightarrow \rightarrow$ ).
}

Vissers et al. (2006) manipulated conflict strength by varying the cloze probability of a misspelled word. In one condition they embedded orthographically incorrect but phonologically correct words (i.e., pseudohomophones) in sentences with a high cloze probability for the critical word (see example 5). In another condition they embedded the misspelled words in sentences with a low cloze probability for the critical word (see example 6).

(5) De kussens zijn opgevuld met verun ... The pillows are stuffed with feathurs ... (literal translation)

(6) Haar walkman deed het niet meer vanwege de verun ... Her walkman did not work anymore because of the feathurs ... (paraphrase)

Although both conditions contained a spelling violation, Vissers et al. (2006) assumed that only in the high cloze probability condition the critical word in its correct spelling was highly expected. Therefore, in this condition the misspelling should elicit a strongly competing representation resulting in a conflict triggering a P600 effect. In the low cloze probability condition the critical word was not expected, and therefore the misspellings were not assumed to elicit such a strong conflict. The results indeed showed a P600 effect for the high cloze probability misspellings only. Note, however, that the sentences in the low cloze probability condition of Vissers et al. (2006) were created by exchanging critical words between high cloze probability sentences. For instance, example 6 was created from the following high cloze probability sentence:

(7) Haar walkman deed het niet meer vanwege de batterijen ... Her walkman did not work anymore because of the batteries ... (paraphrase)

In effect, the sentence contexts in this condition had a low cloze probability for the critical word, but still elicited a high expectation for another word. We hypothesized that this could still have triggered a strong conflict between competing representations of the expected and observed word. An indication in this direction was that Vissers et al. (2006) found correctly spelled critical words in the low cloze probability condition to elicit a biphasic N400-P600 pattern. In a direct comparison between correct and misspelled words, a P600 response to misspellings might thus have been subtracted out. In the present study we, therefore, used a low cloze probability condition in which the sentence context created no expectation for any particular continuation (see example 8).

(8) Op die plek liggen soms verun ... At that spot there sometimes lie feathurs ... (paraphrase)

This cloze probability variation resulted in sentences which were either strongly predictive (high cloze probability) or weakly predictive (low cloze probability) of a certain word, thereby manipulating conflict strength. A stronger representational conflict should be triggered in the case of the high cloze probability misspellings, since the word was expected based on the sentence context as well as incorrectly spelled. For the low cloze probability misspellings the conflict should be weaker, because although the word was misspelled, there was no expectation based on the sentence context.

\section{EEG experiment}

\section{Introduction}

An EEG experiment was conducted to test whether, as the monitoring theory of language perception hypothesizes, the spelling violations indeed elicited the same central-posterior distributed P600 effect compared to the syntactic violations within subjects. A similar scalp topography would support the view that qualitatively similar brain processes are involved in both conditions. In contrast, a difference in scalp topography would indicate that at least partially different processes are engaged in processing syntactic vs. spelling violations. 
In addition, the effectivity of the conflict strength manipulation of the misspellings was tested. Assuming that the representational conflict for the high cloze probability misspellings is stronger, we expected a larger P600 effect for this condition relative to the low cloze probability misspellings.

\section{Materials and methods}

\section{Participants}

Thirty-two healthy right-handed native speakers of Dutch participated in the EEG experiment. Twenty-eight participants were included in the final analyses ( 21 women; mean age $=21.3$ years; age range $=18$ to 26 years). Four participants were excluded because of excessive eye-movement artefacts or apparatus failure. All participants had normal or corrected-to-normal vision, had no language disability, and had no neurological or psychological impairment. The study was approved by the local ethics committee. Written informed consent was obtained prior to participation. The participants were paid or received course credit for their participation.

\section{Stimulus materials}

Spelling violation materials. The spelling violation materials consisted of 116 high cloze probability and 116 low cloze probability sentences. Critical words in the high cloze probability sentences had a mean cloze probability of 0.91 and were identical to those that were used in the study by Vissers et al. (2006). The novel low cloze probability sentence contexts were tested in a pilot study with 15 participants (10 women; mean age $=24.1$ years; age range $=20$ to 29 years) and had a mean cloze probability of 0.10 . The critical low cloze probability words that were used were all plausible continuations of the sentences and had a cloze probability of 0 . The high and low cloze probability sentences were matched for critical word position, length and mean number of clauses.

The critical words were the same in the high and low cloze probability sentences and appeared in mid-sentence position. In different conditions, the critical words were either correctly spelled or misspelled. Spelling violations were created by changing one letter of the word while keeping the phonology the same (see Vissers et al., 2006). This resulted in four sentence conditions: high cloze probability correct, high cloze probability misspelling, low cloze probability correct, and low cloze probability misspelling (see Table 1 for examples).

Sixty filler sentences adapted from Vissers et al. (2006) were used. These consisted of 30 correct sentences, 10 sentences with spelling violations at the beginning, 10 sentences with spelling violations in the middle, and 10 sentences with spelling violations at the end of the sentences.

Eight experimental lists were created on the basis of these materials, which were presented to an equal number of participants.

Table 1

Example of the four sentence conditions of the spelling materials.

\begin{tabular}{ll}
\hline Condition & Sentence \\
\hline $\begin{array}{l}\text { High cloze probability } \\
\text { correct }\end{array}$ & $\begin{array}{l}\text { De kussens zijn opgevuld met veren waardoor ze zacht } \\
\text { aanvoelen. } \\
\text { (The pillows are stuffed with feathers which make them } \\
\text { feel soft.) } \\
\text { De kussens zijn opgevuld met verun waardoor ze zacht } \\
\text { aanvoelen. } \\
\text { migh cloze probabiliting } \\
\text { (The pillows are stuffed with feathurs which make them } \\
\text { feel soft.) }\end{array}$ \\
$\begin{array}{l}\text { Op die plek liggen soms veren van fazanten en pauwen. } \\
\text { (At that spot there sometimes lie feathers from pheasants } \\
\text { and peacocks.) }\end{array}$ \\
$\begin{array}{l}\text { Op die plek liggen soms verun van fazanten en pauwen. } \\
\text { (At that spot there sometimes lie feathurs from pheasants } \\
\text { and peacocks.) }\end{array}$
\end{tabular}

Note: The critical word is underlined and the translation is given in parentheses.
For each critical word the four sentence conditions were counterbalanced across four lists in such a way that participants saw only one condition of a critical word. Each list contained 29 sentences per condition (high cloze probability correct, high cloze probability misspelling, low cloze probability correct, and low cloze probability misspelling). To each list the sixty filler sentences were added. The lists consisted of two blocks ( 88 trials each) that contained an equal amount of sentences per condition. The blocks were matched for critical word position and sentence length. The order of these blocks was reversed to create eight experimental lists in total. Within each block the trials were pseudorandomized using the following constraints: each block began with two filler trials, a filler or experimental trial never occurred more than three times in a row, a spelling violation never occurred more than three times in a row, and a certain condition never occurred more than two times in a row. Furthermore, for both the high and low cloze probability sentences, the correct and misspelling trials were equally often preceded by a correct or misspelling trial.

Syntactic violation materials. Fifty-eight experimental sentences were created. Each sentence had two versions: a syntactically correct and a syntactically incorrect version that contained a number agreement violation on the verb (see Table 2 for an example). Half of the sentences contained an incorrect singular verb and half an incorrect plural verb. Furthermore, half of the subject NPs had animate and half inanimate referents. The mean length, mean position of the critical word, and mean number of clauses were matched to the spelling experimental materials.

A post-hoc cloze test with 16 participants ( 12 women; mean age $=$ 26,4 , age range $=23$ to 31 years) of the experimental sentences of the syntactic materials indicated a medium cloze probability of 0.50 . In this regard the syntactic materials were not matched to the spelling materials for expectancy. Therefore, quantitative direct comparisons between the syntactic and spelling materials will not be conducted.

Thirty filler sentences were created consisting of 15 syntactically correct and 15 syntactically incorrect sentences. The incorrect fillers also contained a number agreement violation on the verb, but compared to the experimental sentences the violations occurred very early in the sentence or later on a second verb. Just as the experimental sentences, the filler sentences were matched to the spelling filler materials for mean length, mean position of the critical word, and mean number of clauses.

On the basis of these materials two experimental lists were created, which were presented to an equal number of participants. The two versions of each sentence were counterbalanced across lists in such a way that participants saw only one version of a sentence. Each list consisted of one block that contained 29 syntactically correct, 29 syntactically incorrect, and 30 filler sentences. The trials were pseudorandomized using the same constraints as for the spelling materials. Syntactically correct and incorrect trials were equally often preceded by a syntactically correct or incorrect trial.

Procedure. The stimuli were presented using the Presentation software (Neurobehavioral Systems, www.neurobs.com). Participants were

Table 2

Example of the two sentence conditions of the syntactic materials.

\begin{tabular}{ll}
\hline Condition & Sentence \\
\hline Syntactically correct & $\begin{array}{l}\text { De schone kleren en handdoeken hangen aan de waslijn te } \\
\text { drogen. } \\
\text { (The clean clothes and towels hang out on the clothesline } \\
\text { to dry.) }\end{array}$ \\
Dyntactically incorrect schone kleren en handdoeken hangt an de waslijn te \\
$\begin{array}{l}\text { De sogen. } \\
\text { (The clean clothes and towels hangs out on the clothesline } \\
\text { to dry.) }\end{array}$
\end{tabular}

Note: The critical word is underlined and the translation is given in parentheses. 
tested individually seated in front of a computer screen. The words were presented in white capitals on a grey background (maximum visual angle $5.2^{\circ}$ ) with a word duration of $350 \mathrm{~ms}$ and a stimulus-onset asynchrony of $645 \mathrm{~ms}$. Trials began with a fixation cross (duration $=510 \mathrm{~ms}$ ) followed by a $500 \mathrm{~ms}$ blank screen. Inter-trial intervals lasted $2000 \mathrm{~ms}$. Participants were instructed to blink between sentences.

To ensure that the participants attentively read the sentences, $10 \%$ of the sentences was followed by a 'yes' or 'no' question about the previous sentence content. The questions were always preceded by a correct sentence condition and followed by a filler trial. The participants had to respond by pressing a button with the left or right index finger. The questions disappeared from the screen when the participants pressed a button or when they failed to respond within $3 \mathrm{~s}$. Questions to which the participants failed to respond within $3 \mathrm{~s}$ were counted as an error.

The experiment was divided into 3 runs ( 2 spelling blocks and 1 syntactic block) that each lasted about $20 \mathrm{~min}$. Pauses were given in between the runs. The order of the blocks was counterbalanced across subjects with the provision that the two spelling blocks always followed each other. This 'blocked design', in which the spelling and syntactic violations were not mixed, was chosen for comparability to our previous studies and because list composition has been found to influence ERP results (e.g., Chwilla et al., 2000).

Data acquisition and analysis. The EEG was recorded continuously with 27 electrodes mounted in an elastic electrode cap (Electro-Cap International). The montage included 5 midline and 22 lateral sites (see Fig. 1). The left mastoid served as a reference. The electro-oculogram (EOG) was recorded by horizontal EOG electrodes with a right to left canthal montage and vertical EOG electrodes placed below and above the right eye. The ground was placed on the forehead, in between both eyes. Electrode impedance was less than $5 \mathrm{k} \Omega$ for the EOG electrodes, and less than $3 \mathrm{k} \Omega$ for the other electrodes. The signals were amplified (time constant $=8 \mathrm{~s}$, band pass $=0.02-30 \mathrm{~Hz}$ ) and digitized on-line with a sampling frequency of $200 \mathrm{~Hz}$.

Before the analyses, the signal was re-referenced to the average of the left and right mastoids. EEG and EOG records were examined for artefacts and excessive EOG amplitude $(>100 \mu \mathrm{V})$ from $100 \mathrm{~ms}$ before the onset of the critical letter string up to $1000 \mathrm{~ms}$ following its onset. Contaminated trials were removed and averages were aligned to a

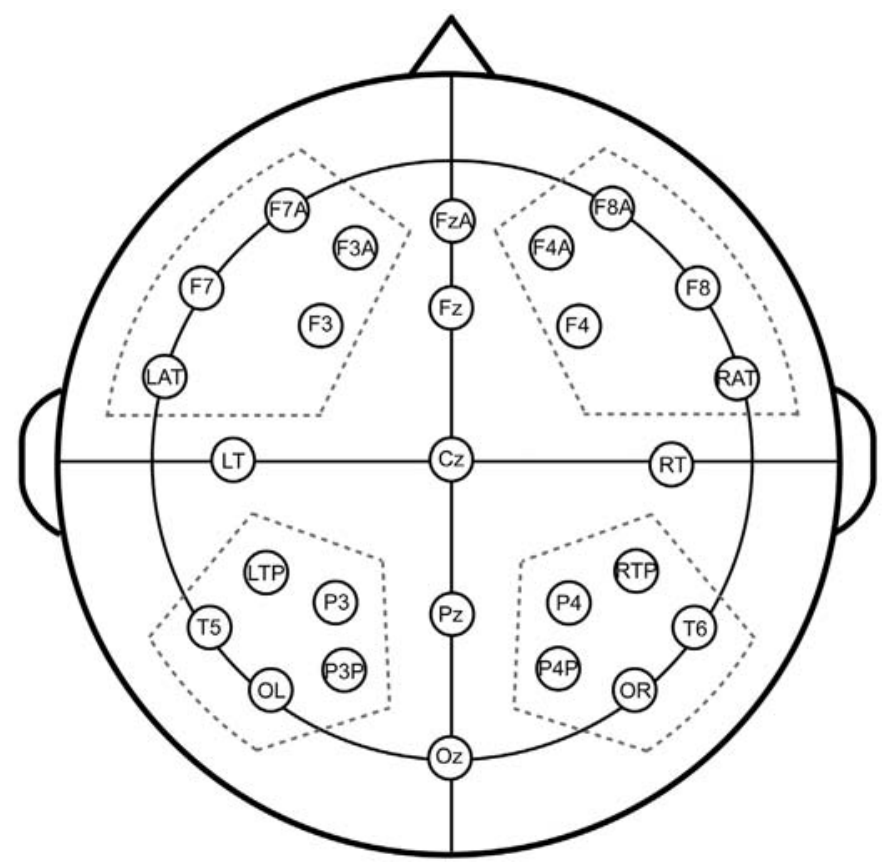

Fig. 1. Electrode montage used in the EEG experiment. 100-ms baseline period preceding the critical letter string. Based upon visual inspection and previous literature, mean amplitudes were calculated in an early (300-500 ms) and late time window (500$800 \mathrm{~ms}$ ) to capture the N400 and P600 effects, respectively.

Repeated measures analyses of variance (MANOVAs) were conducted separately for the midline and lateral sites with Syntax (correct and incorrect) as critical factor for the syntactic data and Spelling (correct and misspelling) and Cloze probability (high and low) as critical factors for the spelling data. Next to these critical factors, the midline analyses included the factor Site (FzA, Fz, Cz, Pz and $\mathrm{Oz}$ ) and the lateral analyses included the factors Region (anterior, posterior), Hemisphere (left, right) and Site. The factors Hemisphere and Region divided the electrodes into four quadrants: left anterior (F7A, F3A, F7, F3 and LAT), left posterior (LTP, P3, P3P, T5 and OL), right anterior (F8A, F4A, F8, F4 and RAT), and right posterior (RTP, P4, P4P, T6 and OR). Interactions with the factor Site were followed up by paired $t$-tests at the single-site level. To avoid problems concerning sphericity, the multivariate approach to repeated measures was used (e.g., Vasey and Thayer, 1987). The report of the ERP results will be restricted to the relevant effects including the critical factor(s).

\section{Results}

Performance on the comprehension task

Mean error rate on the comprehension questions was $10.6 \%$ (syntactic part: 11.5\%; spelling part: 10.1\%). The low error percentages indicate that the participants read the sentences attentively.

\section{Event-related potentials}

The mean percentage of trials that had to be rejected because of artefacts and excessive EOG amplitude was $6.65 \%$ for the syntactically correct and $6.28 \%$ for the syntactically incorrect condition. In the spelling materials this was $8.25 \%$ for the high cloze probability correct, $8.62 \%$ for the high cloze probability misspelling, $4.92 \%$ for the low cloze probability correct, and $5.67 \%$ for the low cloze probability misspelling conditions.

Grand-average waveforms for the syntactic data and the spelling data time-locked to the onset of the critical letter string are presented in Figs. 2 and 3, respectively. All conditions elicited the for visual stimuli characteristic early ERP response - that is, an N1 followed by a P2 which at occipital sites was preceded by a P1. Visual inspection of the waveforms suggested the presence of a P600 effect for the syntactically incorrect vs. syntactically correct, high cloze probability misspelling vs. high cloze probability correct, and low cloze probability misspelling vs. low cloze probability correct conditions, maximal at central-posterior sites. In addition, inspection of the waveforms of the spelling data suggested that an N400 effect was present for the high cloze probability misspelling vs. high cloze probability correct, and low cloze probability misspelling vs. low cloze probability correct conditions at left frontal and temporal sites. Furthermore, a broadly distributed standard cloze probability effect (i.e., a more negative N400 amplitude for the low compared to the high cloze probability condition) with a centro-parietal maximum seemed to be present for both the correct words and the misspellings.

\section{N400 window (300-500 ms)}

No significant results were found within this window for the syntactic data. ${ }^{2}$

\footnotetext{
2 Syntactic violations have been found to elicit an Early Left Anterior Negativity (ELAN; 100-300 ms) or Left Anterior Negativity (LAN; 300-500 ms) (see e.g., Kutas et al., 2006). However, neither in the present study nor in previous ERP studies from our lab in which syntactic violations were investigated (E)LAN effects were observed.
} 


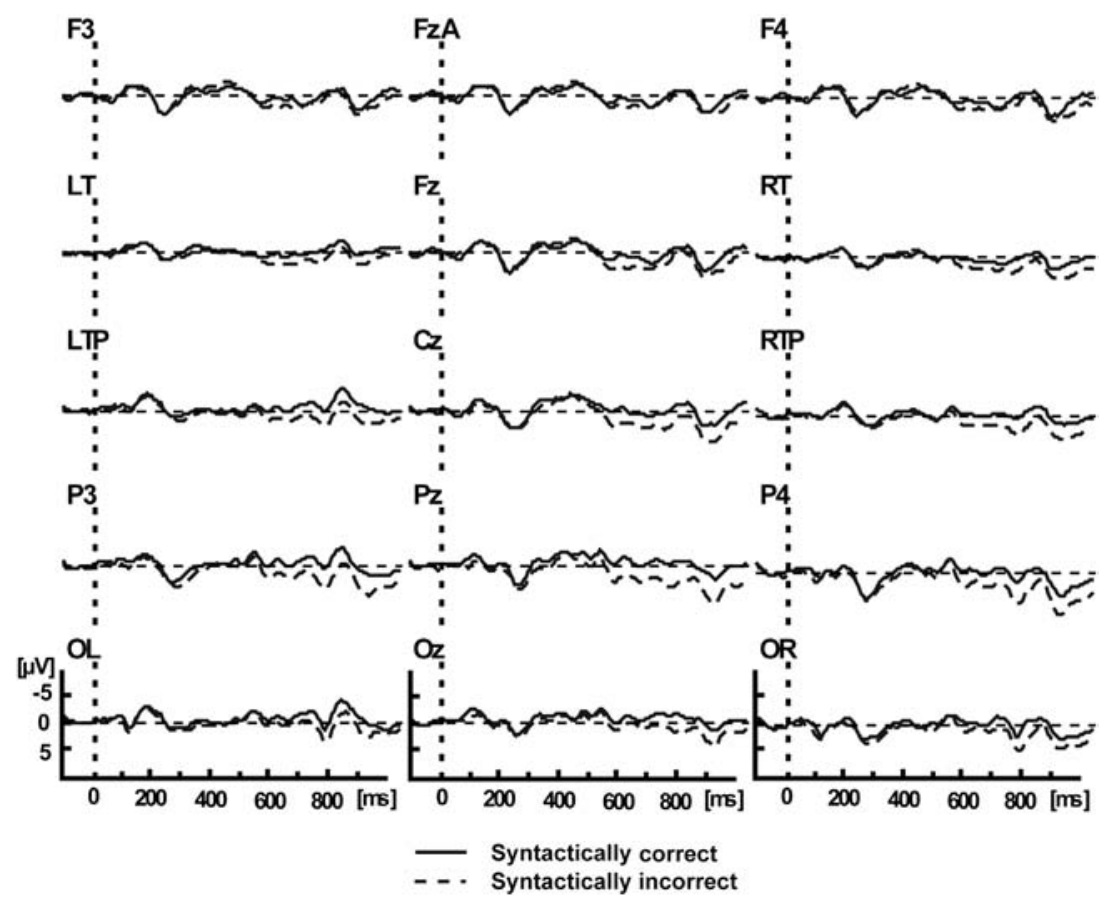

Fig. 2. Grand-average ERP waveforms to the critical words for all midline and a subset of lateral sites, for the syntactically incorrect vs. syntactically correct condition.

For the spelling data, a main effect of Cloze probability for the midline sites $[F(1,27)=60.41, p<0.001]$ and for the lateral sites $[F(1,27)=37.09, p<0.001]$ reflected that overall mean amplitudes were more negative for the low cloze compared to the high cloze probability conditions. For the lateral sites a three-way interaction of Cloze probability $\times$ Spelling $\times$ Hemisphere was present $[F(1,27)=$ $6.06, p<0.05]$. Therefore, follow-up analyses were conducted for the two spelling conditions and the two cloze probability levels separately.
N400 cloze probability effect of words and misspellings

A standard cloze probability effect was found for both the misspelled and the correctly spelled words. The correctly spelled words revealed a main effect of Cloze probability for the midline sites $[F(1,27)=29.00$, $p<0.001]$ and for the lateral sites $[F(1,27)=18.49, p<0.001]$. In addition, Cloze probability $\times$ Site interactions were present [midline sites: $F(4,24)=5.20, p<0.01$, lateral sites: $F(4,24)=8.57, p<0.001]$. For the lateral sites a Cloze probability $\times$ Region $\times$ Site interaction $[F(4,24)=$ 4.92, $p<0.001$ ] and a Cloze probability $\times$ Hemisphere $\times$ Region $\times$ Site

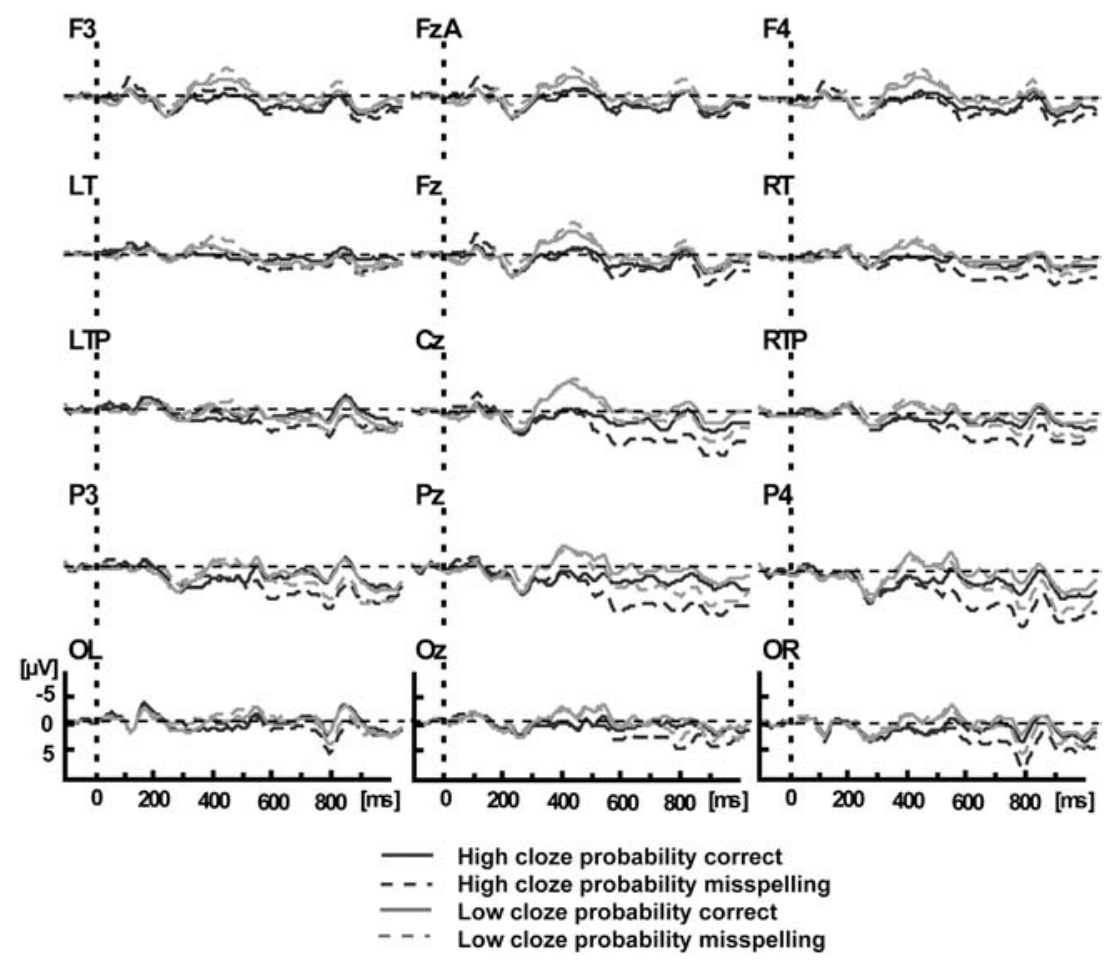

Fig. 3. Grand-average ERP waveforms to the critical words for all midline and a subset of lateral sites, for all spelling conditions. 
interaction $[F(4,24)=3.38, p<0.05]$ were present. Follow-up single site analyses confirmed a broadly distributed N400 effect for the low cloze probability correct condition at all midline and a subset of lateral sites (all $p s<0.05$, except F7A, F8A, F7, F8, LAT and T5).

Likewise, the analysis of the misspellings showed a main effect of Cloze probability for the midline sites $[F(1,27)=51.17, p<0.001]$ and for the lateral sites $[F(1,27)=37.80 p<0.001]$, and a Cloze probability $\times$ Site interaction for both $[F(4,24)=6.95, p<0.001$ and $F(4,24)=$ $6.96, p<0.001$, respectively]. For the lateral sites interactions between Cloze probability $\times$ Hemisphere $[F(1,27)=29.82, p<0.001]$, Cloze probability $\times$ Region $\times$ Site $[F(4,24)=3.69, p<0.01]$ and Cloze probability $\times$ Hemisphere $\times$ Region $\times$ Site $[F(4,24)=3.17, p<0.05]$ were present. Single-site analyses yielded a broadly distributed N400 effect for the low cloze probability misspelling condition at all midline and a subset of lateral sites (all ps. $<0.05$, except F7A, F7 and T5).

\section{N400 effect within the high and low cloze probability conditions}

No significant effects were found for the midline sites. The lateral analyses for the high cloze probability conditions indicated a Spelling $\times$ Hemisphere interaction $[F(1,27)=19.50, p<0.001]$ and a Spelling $\times$ Hemisphere $\times$ Region $\times$ Site interaction $[F(4,24)=3.35$, $p<0.05]$. The interactions reflected that only at the left hemisphere N400 amplitude was larger (i.e., more negative going) for the high cloze probability misspelling compared to the high cloze probability correct condition at F7, LAT, LT, LTP, T5 and OL.

The lateral analysis for the low cloze probability conditions showed a main effect of Spelling $[F(1,27)=7.70, p<0.01]$, reflecting a broadly distributed $\mathrm{N} 400$ effect for the low cloze probability misspelling condition. ${ }^{3}$

\section{P600 window (500-800 ms)}

Syntactic violations. For the syntactic data a main effect of Syntax $[F(1,27)=9.60, p<0.01]$ and a Syntax $\times$ Site interaction $[F(4,24)=$ $4.89, p<0.01]$ were found for the midline sites. This indicated that P600 amplitude was larger (i.e., more positive going) for the syntactically incorrect compared to the syntactically correct condition at central-posterior sites $(\mathrm{Cz}, \mathrm{Pz}$ and $\mathrm{Oz})$. At the lateral sites a main effect of Syntax $[F(1,27)=9.87, p<0.01]$ and a Syntax $\times$ Region interaction $[F(1,27)=9.00, p<0.01]$ were present. Separate analyses of the anterior and posterior region revealed a P600 effect for the syntactically incorrect condition over the posterior region only $[F(1,27)=$ 18.39, $p<0.001]$.

Spelling violations. For the spelling data main effects of Cloze probability and Spelling were found for the midline sites $[F(1,27)=$ 24.59, $p<0.001$ and $F(1,27)=7.83, p<0.01$, respectively] and for the

\footnotetext{
${ }^{3}$ Newman and Connolly (2004) reported an N270 after pseudohomophones and orthographically unexpected words. They proposed that the amplitude of the N270 is modulated by mismatches between the orthographic input and the orthographic expectations elicited by the context. Therefore, following Newman and Connolly (2004) and Vissers et al. (2006) additional analyses were performed on the most negative peak in the 200-350 ms window. These analyses showed an effect of Cloze probability for the midline sites $[F(1,27)=21.76, p<0.00]$ and lateral sites $[F(1,27)=$ $11.71, p<0.01$ ], indicating that the amplitude of the N270 was larger for the low than the high cloze probability conditions. The lateral sites revealed a Cloze probability $\times$ Spelling interaction $[F(1,27)=4.38, p<0.05]$ and Cloze probability $\times$ Spelling $\times$ Hemisphere interaction $[F(1,27)=4.99, p<0.05]$. Analyses of both hemispheres separately revealed a Cloze probability $\times$ Spelling interaction for the right hemisphere only $[F(1,27)=7.25, p<0.05]$. Hence, separate analyses of the right hemisphere were performed for the two levels of cloze. This resulted in an effect of Spelling for the low cloze probability conditions only $[F(1,27)=6.05, p<0.05]$. No interactions with Spelling were obtained. These results indicated a larger N270 amplitude for the low cloze probability misspelling compared to the low cloze probability correct condition across the right hemisphere. The study by Vissers et al. (2006) also showed a larger N270 amplitude to low cloze probability misspellings only. They propose that the misspellings in the high cloze probability condition might not be detected at this early stage due to an 'orthographic illusion' (see Vissers et al., 2006).
}

lateral sites $[F(1,27)=15.08, p<0.001$ and $F(1,27)=6.90, p<0.05$, respectively]. This reflected that overall mean amplitudes were more positive for the high than the low cloze probability conditions and more positive for the misspellings than the correct words. Interactions of Cloze probability $\times$ Spelling were present for the midline sites $[F(1,27)=5.28$, $p<0.05]$ and for the lateral sites $[F(1,27)=7.67, p<0.01]$. In addition, for the lateral sites a Cloze probability $\times$ Spelling $\times$ Hemisphere $\times$ Region interaction was present $[F(1,27)=4.55, p<0.05]$. Separate analyses per quadrant indicated a Cloze probability $\times$ Spelling interaction for the left posterior $[F(1,27)=6.39, p<0.05]$ and right posterior quadrant $[F(1,27)=$ $6.73, p<0.05$ ]. These results revealed that at the midline and bilateral posterior regions the $\mathrm{P} 600$ effect to misspellings was larger in the high than the low cloze probability condition.

Separate analyses of the high and low cloze probability conditions confirmed that the mean P600 amplitude was larger for misspellings than corrects words in both. In the high cloze probability condition, at the midline sites a main effect of Spelling $[F(1,27)=11.30, p<0.01]$ and a Spelling $\times$ Site interaction $[F(4,24)=10.59, p<0.001]$ indicated that P600 amplitude was larger for the high cloze probability misspelling compared to the high cloze probability correct condition at $\mathrm{Cz}, \mathrm{Pz}$ and $\mathrm{Oz}$. For the lateral sites, a main effect of Spelling $[F(1,27)=10.88, p<0.01]$ and interactions between Spelling $\times$ Site $[F(4,24)=6.66, p<0.001]$, Spelling $\times$ Hemisphere $[F(1,27)=6.44, p<0.05]$ and Spelling $\times$ Region $[F(1,27)=16.50, p<0.001]$ reflected that for the high cloze probability misspelling condition a P600 effect was present over all posterior sites (all $p s<0.05$ ). In addition, for the latter condition a negativity was found at a single left frontal electrode (F7A).

For the low cloze probability condition a Spelling $\times$ Site interaction was found for the midline sites $[F(4,24)=7.40, p<0.001]$ and for the lateral sites $[F(4,24)=4.32, p<0.01]$. For the lateral sites interactions between Spelling $\times$ Hemisphere $[F(1,27)=5.53, p<0.05]$, Spelling $\times$ Region $[F(1,27)=14.85, p<0.001]$ and Spelling $\times$ Region $\times$ Site $[F(4,24)=$ 7.37, $p<0.05$ ] were present as well. These results showed that P600 amplitude was larger for the low cloze probability misspelling compared to the low cloze probability correct condition at a subset of posterior sites (Pz, Oz, LTP, P3, P3P, RTP, T6, P4, P4P and OR; ps<0.05). As was the case for the high cloze probability misspelling condition, the low cloze probability misspelling condition showed a negativity at F7A. ${ }^{4}$

Topography of the P600 effects. To compare the scalp distributions of the P600 effect elicited by syntactic and spelling violations two additional MANOVAs were conducted. These MANOVAs included the critical factors Material (syntactic, high cloze probability spelling/low cloze probability spelling) and Acceptability (acceptable and unacceptable). Interactions between Material, Acceptability and Site, Hemisphere or Region would indicate the possibility of differences in scalp distribution between the two kinds of violations. If such an interaction was present we normalized the data according to the McCarthy and Wood (1985) procedure which equalizes the amplitude differences between conditions. ${ }^{5}$

When comparing the syntactic with the high cloze probability spelling materials no interactions were found for the midline sites. For

\footnotetext{
${ }^{4}$ To determine whether the effects found in the N400 window could have caused the results in the P600 window, supplementary analyses were conducted in which the data were aligned between 400 and $500 \mathrm{~ms}$. With these supplementary analyses, essentially the same results as with the original analyses were obtained. In particular both high and low cloze probability misspelling conditions elicited a central-posterior distributed P600 effect compared to their correct controls, and this effect was larger in the high than in the low cloze probability conditions. These supplementary analyses reveal that the pattern of results for the $\mathrm{P} 600$ was not caused by differences in the preceding N400 window.

${ }^{5}$ In recent papers it is debated whether normalization should be used (Urbach and Kutas, 2002; Urbach and Kutas, 2006; Wilding, 2006). Therefore, we report both the results from the non-normalized and the normalized data when there is an interaction in the initial analysis.
} 
the lateral sites there was an interaction of Material $\times$ Acceptability $\times$ Hemisphere $[F(1,27)=8.64, p<0.01]$ and a trend for a Material $\times$ Acceptability $\times$ Region interaction $(p=0.06)$. However, separate analyses of the two hemispheres and regions did not reveal Material $\times$ Acceptability $\times$ Site interactions (all $F s<2$ ). The analyses therefore did not reveal significant differences in the topography of the P600 effect to syntactic and high cloze probability spelling violations.

When comparing the syntactic with the low cloze probability spelling materials, no interactions were found for the midline and lateral sites. For the lateral sites, a trend was present for an interaction between Material $\times$ Acceptability $\times$ Hemisphere $(p=0.054)$, Material $\times$ Acceptability $\times$ Hemisphere $\times$ Site $(p=0.057)$, and Material $\times$ Acceptability $\times$ Region $\times$ Site $(p=0.06)$. To check whether these trends could point at differences in scalp topography the data were normalized. The lateral analysis on the normalized data yielded a trend for a Material $\times$ Acceptability $\times$ Hemisphere interaction $(p=0.053)$. However, separate analyses per hemisphere failed to disclose significant Material $\times$ Acceptability $\times$ Site interactions (both $F s<2$ ). These results therefore do not support that there are topographical differences in P600 effect between the syntactic and low cloze probability spelling violations.

\section{Discussion}

In the EEG experiment we compared the effects of representational conflict due to syntactic violations and misspellings. In addition, we tested our conflict strength manipulation of the misspelling material. We changed the low cloze probability condition of Vissers et al. (2006) to be sure that no conflicting representations could be present based on the sentence context.

First, in agreement with the previous literature, both the syntactic and the spelling violations elicited a central-posterior P600 effect. A comparison of the scalp distribution of these P600 effects did not show significant differences. These results are consistent with the assumption of the monitoring theory of language perception that qualitatively similar processes are involved in the processing of syntactic and spelling violations.

Second, in agreement with Vissers et al. (2006) a P600 effect was found to misspellings in high cloze probability sentences. In contrast, a P600 effect was now also present for misspellings in low cloze probability sentences. Our results indicated that the P600 effect to misspellings was modulated by the conflict strength manipulation because the $\mathrm{P} 600$ effect elicited by high cloze probability misspellings was larger compared to the P600 effect elicited by low cloze probability misspellings. These results call for an adjustment of the conclusions made by Vissers et al. (2006) that only misspellings in sentences in which the related correct word is highly expected elicit a strong enough conflict between competing representations and trigger a P600 effect. It seems that in addition to expectations generated by the sentence context, the word context (i.e., the orthography and phonology of the word) as such also has an effect. In the present low cloze probability condition, no specific expectations were generated by the sentence context. However, still a P600 effect to the low cloze probability misspellings was present, indicating that an expectation based upon the word context alone, could trigger a representational conflict of sufficient size.

A study that also reported a P600 modulation related to expectancy was the study by Coulson et al. (1998). They manipulated the proportion of sentences that contained a syntactic violation. Syntactic violations that were improbable to occur in a block (probability of 20\%) elicited larger P600 amplitudes than probable syntactic violations (probability of $80 \%$ ). Coulson et al. (1998) proposed that the differences in $\mathrm{P} 600$ amplitude reflect the way that individuals update their mental models of the environment which are governed by their expectations. A grammatical violation deviates from what is expected based on daily life, and therefore leads to updating of the mental model. However, although participants might expect a grammatical form by default, a probable syntactic violation block leads them to expect an ungrammatical form. In terms of conflict strength the amplitude decrease for the probable syntactic violations is due to a weaker conflict. Although participants come to expect an ungrammatical form, the expectation for a grammatical form is not completely absent leading to a smaller P600 amplitude.

\section{fMRI experiment}

\section{Introduction}

An fMRI experiment using the same stimuli as in the EEG experiment was conducted. The EEG experiment showed that the syntactic and spelling violations elicited similar P600 effects. Therefore, we were interested to see whether the different representational conflicts would also elicit similar brain area activations, a possible candidate being the IIFG. Furthermore, the EEG experiment showed that the P600 effect to misspellings was modulated by the conflict strength. Therefore, we hypothesized that any brain area that is sensitive to the conflict strength manipulation should differentiate between high and low cloze probability misspellings as well.

\section{Materials and methods}

\section{Participants, stimulus materials and procedure}

In the fMRI experiment, 20 other participants that met the same criteria as the ones in the EEG experiment participated. Sixteen participants were included in the final analyses (12 women; mean age $=22.1$ years; age range $=18$ to 26 years). Four subjects were excluded because of excessive head movements or because they made too many errors on the comprehension questions.

The same materials and procedure as in the EEG experiment were used with the following exceptions: The participants lay in the scanner and saw the stimuli via a mirror attached to the head coil. Furthermore, in the fMRI experiment the length of the inter-trial interval, during which a fixation cross was shown, was jittered between 4300 and $6700 \mathrm{~ms}$ (mean $5500 \mathrm{~ms}$ ), followed by a $500 \mathrm{~ms}$ blank screen. In addition, to avoid left hemisphere motor activation, participants were asked to respond to the content questions with the left index or middle fingers.

Between the first and the second run there was a short break in which the anatomical T1 images were acquired. Between the second and third run the participants were taken out of the scanner for a short break.

\section{Data acquisition and analysis}

The fMRI data were acquired on a $3 \mathrm{~T}$ Siemens Trio scanner. The functional images were obtained using a T2*-weighted EPI-BOLD fMRI scan $\left(\mathrm{TR}=2400 \mathrm{~ms}, \mathrm{TE}=30 \mathrm{~ms}, 80^{\circ}\right.$ flip angle). Thirty-five slices were acquired in an ascending order with a voxel size of $3.5 \times 3.5 \times 3.0 \mathrm{~mm}$ and a field of view of $224 \mathrm{~mm}$. The anatomical images were obtained using a T1-weighted MP-RAGE GRAPPA sequence $\left(\mathrm{TR}=2300 \mathrm{~ms}, \mathrm{TE}=3.03 \mathrm{~ms}, 8^{\circ}\right.$ flip angle, 192 slices, voxel size $=1.0 \times 1.0 \times 1.0 \mathrm{~mm}$, field of view $=256 \mathrm{~mm}$ ).

The fMRI data were preprocessed and analysed using the SPM5 software (http://www.fil.ion.ucl.ac.uk/spm). The first five volumes of each participant were discarded to allow for T1 equilibration effects. The functional images were realigned, slice-time corrected, and each subjects' mean functional image was coregistered to the subjects' anatomical T1 image. Subsequently, the images were anatomically normalized to a T1 template image and smoothed with an $8 \mathrm{~mm}$ FWHM Gaussian kernel.

The data of the spelling and syntactic materials were analysed separately. At the first level, single-subjects analyses were conducted. 
Table 3

MNI coordinates of the ROIs that were selected based on syntactic violation studies.

\begin{tabular}{|c|c|c|c|c|c|}
\hline Study & Violation type & IIFG & rIFG & IPSTG & ACG \\
\hline Cooke et al. (2006) & Inflection, word category, transitivity violations & $\begin{array}{l}{[-61,7,26]} \\
{[-44,11,27]} \\
{[-53,-2,43]}\end{array}$ & $\begin{array}{l}{[48,7,35]} \\
{[48,6,44]}\end{array}$ & & $\begin{array}{l}{[4,14,53]} \\
{[-12,6,57]} \\
{[4,6,57]}\end{array}$ \\
\hline Embick et al. (2000) & Word order violation & {$[-52,10,19]$} & {$[46,17,16]$} & {$[-59,-34,9]$} & \\
\hline Friederici et al. (2003) & Phrase structure violation & & & {$[-62,-42,20]$} & \\
\hline Kang et al. (1999) & Word category violation & $\begin{array}{l}{[-51,15,14]} \\
{[-45,26,6]}\end{array}$ & & & {$[0,0,44]$} \\
\hline Kuperberg et al. (2008) & Number agreement violation & {$[-49,3,8]$} & & {$[-54,-21,-2]$} & \\
\hline Meyer et al. (2000) & Phrase structure, number/gender/case agreement violations & & & {$[-56,-41,5]$} & \\
\hline Moro et al. (2001) & Word order, number agreement violations in pseudoword sentences & & $\begin{array}{l}{[59,22,10]} \\
{[61,14,14]} \\
{[51,14,14]} \\
{[59,22,19]}\end{array}$ & & \\
\hline Nichelli et al. (1995) & n.s. & & {$[40,15,31]$} & & {$[0,16,49]$} \\
\hline Wartenburger et al. (2004) & Word order violation & {$[-60,16,18]$} & & & \\
\hline
\end{tabular}

The linear models of each subject included regressors that modelled the sentence conditions (syntactically correct and incorrect; high cloze probability correct and misspelling, low cloze probability correct and misspelling) from the critical word onwards to the end of the sentence. The correct filler sentences and the correct experimental sentence beginnings (until the word before the critical word) were included in a 'general correct' (GC) regressor. In addition, regressors were included for the inter-trial interval in which a fixation was shown (FIX) and for six realignment parameters describing head movements. The regressors were convolved with a canonical hemodynamic response function. For the second-level analysis, the contrast images from the first level were entered into a random effects group analysis.

Whole brain analysis. In the whole brain analysis of both the syntactic and spelling data we obtained the single-subject contrast images for all experimental conditions and GC relative to FIX. Differential effects of the experimental conditions were calculated at the second-level with a full factorial design for both the syntactic and the spelling data. The results of the analyses were thresholded at $p<0.001$ (uncorrected). Cluster size was used as a test-statistic, and only activation clusters at a threshold of $p<0.05$ (corrected) are reported. All local maxima are reported as MNI coordinates.

Region of interest specification. To test for possible material specific activation in the fMRI experiment, we selected regions of interest (ROIs) based on two different sets of studies. First, we conducted a meta-analysis of twelve neuroimaging studies that contained syntactic violations, and included an area as a ROI when three or more studies reported this area (see Table 3). ${ }^{6}$ Four ROIs were selected based on these studies: left inferior frontal gyrus (IIFG), right inferior frontal gyrus (rIFG), left posterior superior temporal gyrus (IPSTG), and anterior cingulate gyrus (ACG). The mean coordinates and mean distance of the local maxima to the mean coordinates were calculated. The radius of the ROIs was based on these mean distances, resulting in the following mean coordinates and spheres: IIFG [-52, 11, 21], $12 \mathrm{~mm}$ sphere; rIFG [52, 15, 23], $14 \mathrm{~mm}$ sphere; IPSTG [-58, - 35, 8], $10 \mathrm{~mm}$ sphere; ACG $[-1,8,52], 9 \mathrm{~mm}$ sphere (see Fig. 4 for a depiction of the ROIs).

Second, since at present few neuroimaging studies have been conducted using pseudohomophones, we based our ROIs for the spelling violations on a meta-analysis on pseudowords by Mechelli et al. (2003). Three ROIs were selected based on this meta-analysis: left inferior temporal gyrus (IITG), IIFG, and right cerebellum. Again the

\footnotetext{
${ }^{6}$ Only nine studies are included in Table 3 because the other three (Indefrey et al., 2001; Kuperberg et al., 2003a, 2000) did not find activation in the ROIs and/or did not report coordinates.
}

mean coordinates and distance were calculated (IITG $[-47,-62,-20]$, $9 \mathrm{~mm}$ sphere; IIFG [ $-45,12,14], 14 \mathrm{~mm}$ sphere; right cerebellum [18, -62, - 39], $22 \mathrm{~mm}$ sphere) (see Fig. 4).

Region of interest analysis. An ROI analysis on the syntactic and spelling data was performed in which all the regions were tested using the Marsbar toolbox for SPM (Brett et al., 2002, http://marsbar. sourceforge.net). The lIFG region resulting from the syntactic studies $\left(\mathrm{IIFG}_{\text {syn }}\right)$ and the IIFG region resulting from the pseudoword studies $\left(\mathrm{IIFG}_{\text {spel }}\right)$ were tested in separate analyses because they showed some overlap.

A $2 \times 6$ repeated measures MANOVA was performed on the syntactic data, including the factor Syntax (correct, incorrect) and

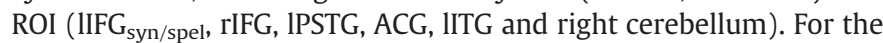
spelling data a $2 \times 2 \times 6$ repeated measures MANOVA was conducted, including the factor Spelling (correct and misspelling), Cloze

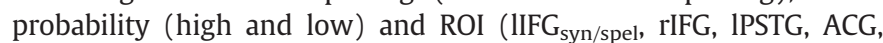
IITG and right cerebellum). These MANOVAs were based on the contrast values that were obtained in the ROIs from the single-subject contrast images for the experimental conditions (syntactically correct, syntactically incorrect; high cloze probability correct, high cloze probability misspelling, low cloze probability correct and low cloze probability misspelling) with the inter-trial interval (FIX) as a baseline.

\section{Results}

Performance on the comprehension task

Mean error rate on the comprehension questions was $9.5 \%$ (syntactic part: 5.6\%; misspelling part: $11.5 \%$ ). Two participants were excluded from the analysis because they made more than 7 errors $(25.9 \%)$ in total. The low error percentages indicate that the participants read the sentences attentively.

\section{Whole brain analysis}

Syntactic data. The results of the contrast syntactically correct>syntactically incorrect are listed in Table 4a. The clusters that were significantly stronger activated for the syntactically correct than syntactically incorrect condition included regions in the anterior and middle cingulate cortex and the right medial frontal gyrus. The contrast syntactically incorrect $>$ syntactically correct yielded no significant results.

Spelling data. In the whole brain analysis of the spelling data a comparison was made between the misspelled and correctly spelled conditions. The contrast correctly spelled $>$ misspelled yielded no significant results. The results of the contrast misspelled $>$ correctly spelled are listed in Table $4 \mathrm{~b}$. Regions that were significantly stronger activated for the misspelling conditions compared to the correctly 

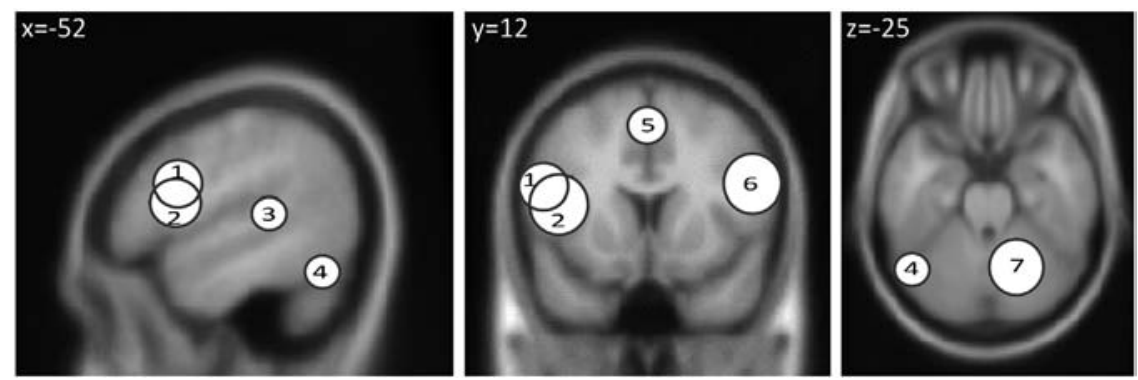

Fig. 4. ROIs used in the present study, $1=\mathrm{IIFG}_{\text {syn }} ; 2=\mathrm{IIFG}_{\text {spel }} ; 3=\mathrm{IPSTG} ; 4=\mathrm{IITG} ; 5=\mathrm{ACG} ; 6=$ rIFG; $7=$ right cerebellum.

spelled conditions included the right superior occipital gyrus, right superior parietal lobule, left cerebellum and left fusiform gyrus (see Fig. 5a).

In addition, a comparison was made between the high and low cloze probability conditions. In Table $4 \mathrm{c}$ the results of the high cloze probability $>$ low cloze probability contrast are listed. The left and right IPL showed a significantly stronger activation for the high cloze probability conditions (see Fig. 5b). No significant results were obtained with the low cloze probability $>$ high cloze probability contrast.

No significant results were obtained for the interaction of Spelling $\times$ Cloze probability.

\section{Region of interest analysis}

Syntactic data. The analysis including IIFG $_{\text {syn }}$ revealed a Syntax $\times-$ ROI interaction $[F(5,11)=6.30, p<0.01]$. Follow-up analyses showed a main effect of Syntax in $\mathrm{LIFG}_{\text {syn }}$ only $[T(15)=-3.49, p<0.01]$, indicating that the syntactically incorrect condition elicited significantly more activation in IIFG than the syntactically correct condition (mean contrast value syntactically incorrect $=0.73$, syntactically correct $=0.51$, see Fig. 6 )

When using IIFG $_{\text {spel }}$ instead of IIFG $_{\text {syn }}$ in the analysis the same results were obtained; significant results were obtained for IIFG $_{\text {spel }}$ only $[\mathrm{T}(15)=-2.78, p<0.05]$ (mean contrast value syntactically incorrect $=0.43$, syntactically correct $=0.31$, see Fig. 6 ).

Spelling data. The analysis revealed a main effect of Spelling $[F(1,15)=$ 15.39, $p<0.01]$ and a Spelling $\times$ ROI interaction $[F(5,11)=3.92, p<0.05]$.

Table 4

Significant clusters at the whole brain level.

\begin{tabular}{|c|c|c|c|c|c|c|}
\hline Region & BA & Cluster size & $\begin{array}{l}\text { Voxel } \\
\text { T value }\end{array}$ & $x$ & $y$ & $z$ \\
\hline \multicolumn{7}{|c|}{ a. Syntactically correct $>$ syntactically incorrect } \\
\hline \multirow[t]{3}{*}{ Anterior cingulate cortex } & 24 & 631 & 5.28 & 2 & 38 & 4 \\
\hline & 25 & & & 6 & 18 & -4 \\
\hline & & & & -2 & 18 & -2 \\
\hline Right middle cingulate cortex & 9 & 323 & 4.94 & 12 & 30 & 36 \\
\hline \multirow[t]{2}{*}{ Right middle frontal gyrus } & & & & 24 & 32 & 38 \\
\hline & & & & 28 & 26 & 32 \\
\hline \multicolumn{7}{|l|}{ b. Misspelled $>$ correctly spelled } \\
\hline Right superior occipital gyrus & 19 & 476 & 4.62 & 28 & -68 & 48 \\
\hline Right superior parietal lobule & 7 & & 4.57 & 34 & -62 & 54 \\
\hline \multirow[t]{2}{*}{ Left cerebellum } & & 344 & 4.28 & -32 & -74 & -32 \\
\hline & & & 3.68 & -48 & -56 & -34 \\
\hline Left fusiform gyrus & 37 & & 3.62 & -48 & -58 & -22 \\
\hline \multicolumn{7}{|l|}{ c. High $>$ low cloze probability } \\
\hline \multirow[t]{2}{*}{ IIPL } & 40 & 395 & 4.11 & -42 & -60 & 54 \\
\hline & & & 3.89 & -56 & -48 & 42 \\
\hline \multirow[t]{2}{*}{ rIPL } & 40 & 257 & 3.86 & 48 & -54 & 44 \\
\hline & & & 3.67 & 38 & -58 & 46 \\
\hline
\end{tabular}

Note: The table shows all clusters at a significance level of $p<0.05$ corrected at the cluster level (thresholded at $p<0.001$ uncorrected). All local maxima are reported as MNI coordinates.
No main effect of Cloze probability or interaction with this factor was found. Follow-up analyses showed that all ROIs except IPSTG showed a main effect of Spelling (all ps<0.05). This indicated that in IIFG $_{\text {spel }}$, rIFG, IITG, ACG, and right cerebellum the misspelling condition elicited significantly more activation than the correctly spelled condition. When using IIFG $_{\text {syn }}$ in the analysis the same results were obtained; all ROIs except IPSTG showed a main effect of Spelling (all $p s<0.05$ ). Fig. 6 gives an overview of the mean contrast values per ROI.

\section{Discussion}

We investigated whether the representational conflicts elicited by syntactic and spelling violations would generate co-localized activation. In addition, we were interested whether the effects of conflict strength manipulation on the processing of spelling violations that were observed in the EEG experiment would also modulate the hemodynamic response in the fMRI

The results from the ROI analyses showed that both the syntactic and the spelling violations elicited stronger activation in the IIFG relative to their controls. The high and low cloze probability misspellings did not elicit differential activation in this area, indicating no modulation by conflict strength. In addition to the IIFG activation, the spelling violations elicited stronger activation compared to their correct controls in the rIFG, right superior occipital and parietal areas, right and left cerebellum, IITG/left fusiform gyrus, and ACG. Again, the activation in these areas was not modulated by conflict strength.
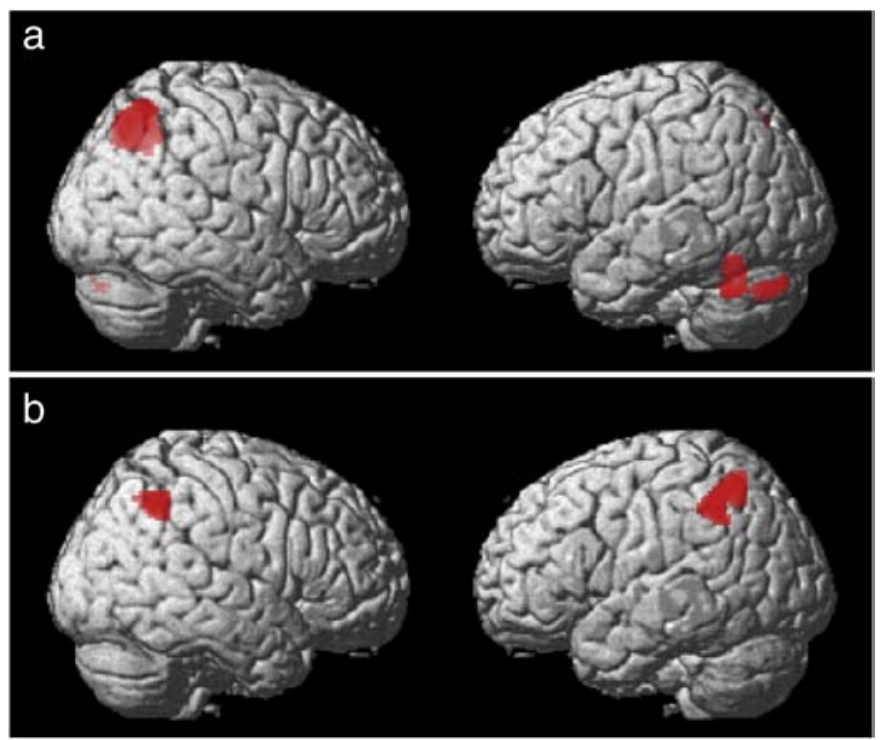

Fig. 5. (a) Significant activation clusters from the whole brain analysis for the misspelled relative to the correctly spelled condition. (b) Significant activation clusters from the whole brain analysis for the high cloze probability relative to the low cloze probability condition. 


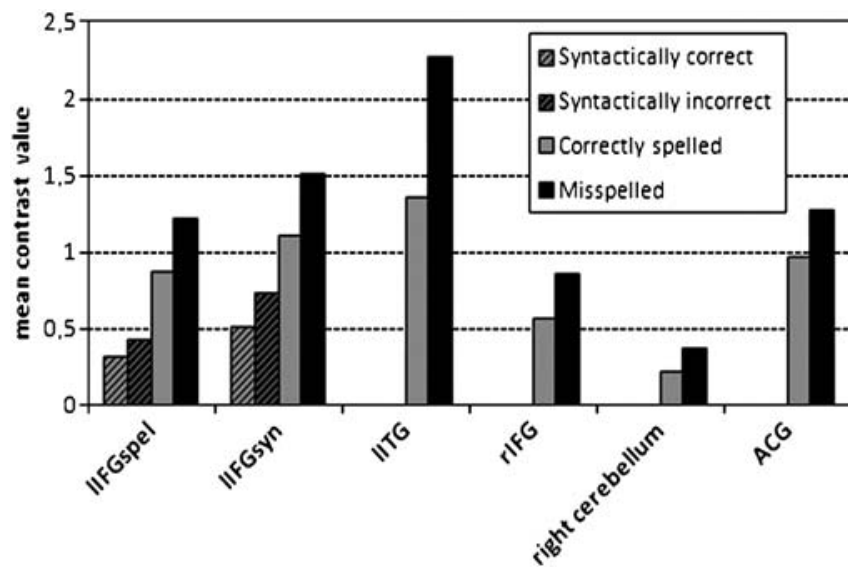

Fig. 6. Mean contrast values per ROI for the syntactically correct and incorrect condition, and the correctly spelled and misspelled condition.

However, the activation was specific to the spelling violations; the syntactic violations did not elicit any other additional activations than the activation in IIFG. In the general discussion we will elaborate on these findings.

Although the ROI analyses revealed significant activation in the IIFG for both types of violations, the whole brain analyses did not. Previous studies, however, did find IIFG modulation at the whole brain level when contrasting for example syntactically incorrect with syntactically correct stimuli (see e.g., Table 3). The fact that we did not find IIFG activation at the whole brain level could be because our experiment did not involve an explicit judgment task, which might increase the underlying neural response. For example, all studies that were included in our meta-analysis of syntactic violation studies that reported IIFG activation at the whole brain level used such a task, except from the study by Kang et al. (1999). We explicitly chose not to use a judgment task, to stay as close as possible to 'normal reading' and because this type of task has been shown to modulate the P600 (e.g., Kuperberg, 2007).

\section{General discussion}

The present study tried to further connect the existing literature on the involvement of cognitive control in dealing with representational conflicts to the literature on error monitoring in language perception. To this end, an EEG and fMRI experiment were conducted investigating representational conflicts created by syntactic and spelling violations. These violations elicited similar ERP responses in previous studies and we were interested whether they would create co-localized activation in the fMRI. The conflict strength created by misspellings was manipulated as well to address the question whether this would modulate both the P600 component and the hemodynamic response. We will first discuss the results of the EEG experiment, then go into the results of the fMRI experiment, and end with a discussion of the differences in findings.

\section{EEG findings}

Previous EEG studies found that syntactic and non-syntactic conflicts elicited positivities with very similar scalp distributions. The present EEG experiment added support to these findings by showing within subjects that both the syntactic and spelling violations elicited a similarly distributed central-posterior P600 effect compared to their correct controls. This suggests that similar processes are involved in the processing of syntactic and spelling violations.

As mentioned briefly in the general introduction, the functional significance of the P600 effect is debated. The dominant view has been that it indexes syntactic processing. For example, it has been proposed that the P600 effect reflects syntactic reanalysis or repair (e.g., Friederici et al., 1996) or syntactic integration difficulties (e.g., Kaan et al., 2000). Hagoort (2003, 2009) interpreted the P600 effect in light of the Unification Model as proposed by Vosse and Kempen (2000). According to this model every incoming word is associated with a lexical frame (i.e., an elementary syntactic tree) that is retrieved from memory. These frames incrementally enter a unification space where they are bound together into a phrasal configuration. This process is dynamic, because due to competing alternative binding candidates the strength of the unification links vary over time until a stable configuration is reached. Hagoort $(2003,2009)$ proposed that the P600 reflects the formation of unification links. The P600 amplitude is thought to be related to the time that is needed to form unification links of sufficient strength, which is influenced by syntactic complexity, by syntactic ambiguity, and by semantic influences.

A different explanation of the P600 effect was given by Kuperberg (2007). She proposed that comprehension of verb-argument relationships within sentences proceeds along at least two interactive processing streams. In the first stream the evolving representation of meaning is evaluated and compared with patterns of relationships that are prestored within semantic memory. The second stream is a combinatorial stream that involves combining words based on multiple rule-like constraints, including morphosyntactic and thematic-semantic constraints, to build up a propositional meaning. According to this account, the P600 effect reflects a continued analysis within the combinatorial stream triggered by a conflict between its output and the output of the semantic memory-based stream.

Although these accounts of the functional significance of the P600 effect, do not predict an effect for spelling violations in and of themselves, they cannot be excluded completely. For example, Kuperberg (2007, p.41) leaves open the question of whether an orthographic processing stream exists. If an orthographic processing stream exists, a P600 effect elicited by a spelling violation could result from a continued analysis within such a stream. From a syntactic or a unification perspective, spelling violations could affect syntactic processing or influence the time needed to establish sufficiently strong unification links. However, we think a syntactic or a unification account is less plausible in the present study because we used pseudohomophones that could easily be traced back to the words from which they were derived. Therefore, we think it is less likely that they would make syntactic processing more difficult. It is also less likely that the time needed to establish sufficiently strong unification links is affected. Since the correct word can easily be traced back, the lexical frames should be accessible and unification should be possible. If it were the case that the pseudohomophones do not lead to the retrieval of lexical frames in memory, we think the Unification Model would predict an (E)LAN. Hagoort $(2003,2009)$ proposed that an (E)LAN results from a binding failure when there is no matching category node. If a pseudohomophone is not associated with a lexical frame, an NP node in the syntactic tree of the phrasal configuration cannot be filled.

The monitoring theory of language perception can account for the finding that both syntactic and spelling violations trigger a similar P600 effect. According to this theory a P600 is elicited when there is a strong representational conflict between an expected and observed element that triggers reprocessing of the input to check for possible processing errors (e.g., Van de Meerendonk et al., 2009). Such a representational conflict is created by both syntactic and spelling violations. For the syntactic violations a conflict arises between an expected grammatical form and an observed ungrammatical form. The spelling violations create a conflict between an expected correctly spelled word and the observation of an incorrectly spelled word.

The monitoring theory assumes that only representational conflicts that are sufficiently strong should trigger reprocessing of the input and elicit a P600. The present EEG experiment manipulated conflict strength by varying expectancy based on the sentence context 
in the spelling materials. The results indicated that the P600 effect was larger for high cloze probability misspellings than low cloze probability misspellings. Our finding of a modulation by conflict strength reinforces the importance of the conflict concept, a notion that is present in other theories of the P600 component as well (see Hagoort et al., 2009; Kuperberg, 2007).

\section{fMRI findings}

The fMRI results showed that syntactic and spelling violations both increased activation in the IIFG compared to their correct controls. This corresponds to the findings of Embick et al. (2000) who compared syntactic word order violations and spelling errors in sentences (e.g., paper written as papger) to a control condition in which rows of coloured letters were shown. In this study, the participants' task was to indicate whether one or two errors were present in the sentences, or whether one or two matches (i.e., a colour-letter combination) occurred in the control condition. Their results also showed that both syntactic and spelling violations elicited increased activation in the IIFG compared to their control condition.

At present, various accounts on the role of the IIFG in language processing exist. For example, increased activation in the IIFG might reflect the computation of syntactic movement of elements in a sentence (e.g., Grodzinsky and Friederici, 2006). Another proposal is that the IIFG activation reflects increased demands on syntactic working memory due to the temporal maintenance of unintegrated syntactic information (e.g., Fiebach et al., 2005). Hagoort (2005) proposed that the IIFG is involved in the unification of lexical elements that are retrieved mainly from the temporal lobes. According to this proposal the IIFG is involved in the syntactic as well as semantic and phonological unification of lexical elements, with a certain level of specialization in different subregions. These different accounts, however, have difficulty in explaining the co-localized IIFG activation for syntactic and spelling violations. The syntactic accounts would have to assume that misspellings affected the syntactic processing, which we think again is less likely since we used pseudohomophones. Unification should not be affected either, because pseudohomophones should not lead to difficulties with the formation of syntactic, semantic or phonological representations.

Novick et al. (2005) proposed that the IIFG is involved in implementing cognitive control to resolve representational conflicts (see also, e.g., Thompson-Schill et al., 2005, 1997). Instead of reflecting a language specific process, it is thought that the process in the IIFG reflects a general conflict resolution mechanism. This account can explain the finding of co-localized IIFG activation for syntactic and spelling violations. Both types of violations create a representational conflict, and when such a conflict is present, the IIFG is thought to guide the neural activity in the appropriate pathways to prevent misinterpretations (e.g., Miller and Cohen, 2001).

Since our EEG results showed a sensitivity to the conflict strength manipulation, we hypothesized that any brain area that is sensitive to this manipulation should differentiate between high and low cloze probability misspellings as well. However, our fMRI results did not show such a differential effect in any brain region, indicating that the hemodynamic response was not modulated by conflict strength. January et al. (2009) also manipulated conflict strength between competing representations in an fMRI study. This was done by using different pairings of pictures and syntactically ambiguous sentences. Hereby, they created a weak (syntactically unambiguous sentences), medium and strong conflict condition. The conflict increased due to the fact that the pictures increasingly supported a less preferred interpretation of the ambiguous sentence (see January et al., 2009 for the specific details). Their results showed a significantly stronger activation in the IIFG for the strong compared to the weak conflict condition only. This result could indicate that two conditions that both create a representational conflict but that differ in their actual conflict strength can differentially activate the IIFG. However, it is unclear whether the weak conflict condition would also show differential effects in the IIFG compared to a no-conflict condition, since this was not tested. In addition, because the conflict manipulation that was used by January et al. (2009) is very different from the manipulation in the present study, it is not clear how their results should be mapped onto the present ones.

In addition to increased activation in the IIFG, the spelling violations also showed increased activation in more posterior areas when compared to their correct controls. These activations could reflect the actual (re)processing of the spelling violations. For example, the IITG/left fusiform gyrus closely corresponded to the visual word form area (VWFA), an area which is thought to compute structural representations of words from abstract letter representations (e.g., Dehaene et al., 2002). It is likely that upon encountering a spelling violation, (re)processing of the visual word form is done, and as indicated by our meta-analysis several studies have found this area to be more activated by pseudowords than words (see Mechelli et al., 2003). Furthermore, the activation that was found in the right occipital area might be related to some extra visual processing of the spelling violation. In contrast, an area that could be involved not in the (re)processing of the spelling violations as such but in conflict processing is the ACG. The ACG has been implicated in conflict detection and it has been suggested that it sends this information to frontal brain areas (e.g., Yeung et al., 2004). Note, however, that we do not find a modulation of the hemodynamic response depending on conflict strength in this region either.

For the syntactic violations, besides the increased activation in IIFG, we did not find any other additional activation. An area that is also often reported to show increased activation to syntactic violations is the IPSTG. First, it could be that our syntactic violations did not elicit a detectable response in the IPSTG because participants were not asked to judge grammatical acceptability of the sentences. All studies that were included in our meta-analysis conducted such a judgment, except the study by Kang et al. (1999) which also did not report activation in the IPSTG. Second, it could be that no IPSTG activation was detected because number agreement violations are relatively subtle compared to other types of violations, like word category violations. Kuperberg et al. (2008), however, did find IPSTG activation to number agreement violations.

\section{Differences EEG and fMRI findings}

It is important to note that we do not want to infer that the IIFG is the underlying neural source of the P600 component. One has to be cautious with such an interpretation because ERP and fMRI measurements capture neural responses at very different time scales. While ERPs allow for the detection of neural responses to critical words, the hemodynamic responses in the fMRI also reflect additional processes that occur after the critical words.

This difference in temporal resolution between ERP and fMRI measurements might explain why our conflict strength manipulation modulated the $\mathrm{P} 600$ but did not affect the hemodynamic response. Since fMRI measurements are temporally less sensitive than ERPs this could have resulted in a null finding for the conflict strength manipulation. In general, null results have to be interpreted cautiously, and they leave open the possibility that the hemodynamic response can be modulated by conflict strength.

Despite of this, we would like to suggest as a post-hoc explanation, that the ERP and fMRI measurements might capture different parts of the monitoring process. Whereas the $\mathrm{P} 600$ amplitude could primarily be related to the strength of the conflict, the hemodynamic response which was not modulated by conflict strength, could indicate the presence of reprocessing. Reprocessing is thought to occur when a conflict is of sufficient size, but would be identical for spelling violations in the high and low cloze probability conditions. This seems plausible, since the violations were identical in both conditions; only 
one letter was changed compared to the correctly spelled words. This does not mean that differences in the degree of reprocessing could not exist. If we would have different degrees of ill-formedness, contrasting for example one-letter-changed-misspellings with misspellings in which more letters are changed, this might result in differences in the amount of reprocessing. It is therefore assumed that the degree of reprocessing is not proportional to the degree of conflict (the conflict just needs to pass a certain 'threshold' to trigger reprocessing), but rather to the degree of ill-formedness of the encountered word.

Under this post-hoc hypothesis, the IIFG would guide the reprocessing of the unexpected representation by guiding activation in more posterior areas (e.g., the IITG/left fusiform gyrus). This is somewhat different from the proposal by Novick et al. (2005) regarding the implementation of cognitive control by the IIFG for conflict resolution in garden-path sentences. To prevent misinterpretations in garden-path sentences, Novick et al. (2005) proposed that selecting the relevant parse involved both the suppression of the initially expected but irrelevant parse and the recovery of the unexpected but relevant parse from all the information present. Our data, however, seem to suggest that the P600 amplitude modulation is related to the suppression of the expected representation, whereas the IIFG activity may correspond to the guidance of the reprocessing (recovery) of the unexpected representation (i.e., the violation) only. The finding that there is a larger P600 amplitude in the high relative to the low cloze probability misspellings, could reflect that more suppression of the expected representation is needed in the high cloze probability condition for which this representation is stronger. This would fit with previous proposals that positivities in the EEG could indicate inhibition of cortical neuronal networks (e.g., Coenen, 1995; Rockstroh et al., 1992, 1996). However, it should be noted that even if the amplitude difference of the P600 in the present study is related to the amount of suppression this does not exclude that the process underlying the P600 does also encompasses some reprocessing.

The possibility that the ERP and fMRI measurements capture different parts of the monitoring process could also explain why we found similarly distributed P600 effects to syntactic and spelling violations, while the hemodynamic response to spelling violations showed that additional regions were engaged. If the P600 effect would reflect an aspect of conflict processing (e.g., suppression of the expected representation) we suppose this process to be similar for syntactic and spelling violations. In contrast, if as proposed previously, the hemodynamic activation would reflect the reprocessing, this might involve brain regions more specific to spelling violations (e.g., the IITG/left fusiform gyrus) than to syntactic violations and vice versa.

\section{Conclusion}

To conclude, the present study further explored representational conflicts underlying the detection and reprocessing of errors in language perception. In accordance with the monitoring theory of language perception, our results show that conflicts elicited by syntactic and spelling violations trigger similar P600 effects, which can be modulated by conflict strength. In addition, the finding of increased IIFG activation for both violation types connects the existing literature of the involvement of IIFG in dealing with representational conflicts to the literature on error monitoring in language perception.

\section{Acknowledgments}

Portions of this research were presented at the 17th Annual Meeting of the Cognitive Neuroscience Society in Montréal, 2010, and at the 7th Forum of European Neuroscience in Amsterdam, 2010. We thank two anonymous reviewers for their helpful comments on previous versions of this article. We are also grateful to the technical group of the Donders Institute for Brain Cognition and Behaviour for their support.

\section{References}

Brett, M., Anton, J.-L., Valabregue, R., Poline, J.-B., 2002. Region of interest analysis using an SPM toolbox. 8th International Conference on Functional Mapping of the Human Brain, Sendai, Japan.

Coenen, A.M.L., 1995. Neuronal activities underlying the electroencephalogram and evoked potentials of sleeping and waking: Implications for information processing. Neurosci. Biobehav. Rev. 19, 447-463.

Chwilla, D.J., Kolk, H.H.J., Mulder, G., 2000. Mediated priming in the lexical decision task: evidence from event-related potentials and reaction time. J. Mem. Lang. 42, 314-341.

Cooke, A., Grossman, M., DeVita, C., Gonzalez-Atavales, J., Moore, P., Chen, W., Gee, J., Detre, J., 2006. Large-scale neural network for sentence processing. Brain Lang. 96 , 14-36.

Coulson, S., King, J.W., Kutas, M., 1998. Expect the unexpected: event-related brain response to morphosyntactic violations. Lang. Cogn. Process. 13, 21-58.

Dehaene, S., Le Clec'H, G., Poline, J.-B., Le Bihan, D., Cohen, L., 2002. The visual word form area: a prelexical representation of visual words in the fusiform gyrus. NeuroReport $13,321-325$.

Embick, D., Marantz, A., Miyashita, Y., O'Neil, W., Sakai, K.L., 2000. A syntactic specialization for Broca's area. Proc. Natl Acad. Sci. USA 97, 6150-6154.

Eriksen, B.A., Eriksen, C.W., 1974. Effects of noise letters upon identification of a target letter in a nonsearch task. Percept. Psychophys. 16, 143-149.

Fiebach, C.J., Schlesewsky, M., Lohmann, G., von Cramon, D.Y., Friederici, A.D., 2005. Revisiting the role of Broca's area in sentence processing: syntactic integration versus syntactic working memory. Hum. Brain Mapp. 24, 79-91.

Friederici, A.D., Pfeifer, E., Hahne, A., 1993. Event-related brain potentials during natural speech processing: effects of semantic, morphological and syntactic violations. Cogn. Brain Res. 1, 183-192.

Friederici, A.D., Hahne, A., Mecklinger, A., 1996. Temporal structure of syntactic parsing: early and late event-related brain potential effects. J. Exp. Psychol. Learn. Mem. Cogn. 22, 1219-1248.

Friederici, A.D., Ruschemeyer, S.-A., Hahne, A., Fiebach, C.J., 2003. The role of left inferior frontal and superior temporal cortex in sentence comprehension: localizing syntactic and semantic processes. Cereb. Cortex 13, 170-177.

Ganushchak, L.Y., Schiller, N.O., 2010. Detection of speech errors in the speech of others an ERP study. Neuroimage 49, 3331-3337.

Grodzinsky, Y., Friederici, A.D., 2006. Neuroimaging of syntax and syntactic processing. Curr. Opin. Neurobiol. 16, 240-246.

Hagoort, P., 2003. How the brain solves the binding problem for language: a neurocomputational model of syntactic processing. Neuroimage 20, S18-S29.

Hagoort, P., 2005. On Broca, brain, and binding: a new framework. Trends Cogn. Sci. 9, $416-423$.

Hagoort, P., 2009. Reflections on the neurobiology of syntax. In: Bickerton, D. Szathmáry, E. (Eds.), Biological Foundations and Origin of Syntax. The MIT Press, Cambridge, MA, pp. 279-296.

Hagoort, P., Brown, C., Groothusen, J., 1993. The Syntactic Positive Shift (SPS) as an ERP measure of syntactic processing. Lang. Cogn. Process. 8, 439-483.

Hagoort, P., Baggio, G., Willems, R.M., 2009. Semantic unification, In: Gazzaniga, M.S. (Ed.), The Cognitive Neurosciences, 4th ed. MIT Press, Cambridge, MA, pp. 819-836.

Hoeks, J.C.J., Stowe, L.A., Doedens, G., 2004. Seeing words in context: the interaction of lexical and sentence level information during reading. Cogn. Brain Res. 19, 59-73.

Indefrey, P., Hagoort, P., Herzog, H., Seitz, R.J., Brown, C.M., 2001. Syntactic processing in left prefrontal cortex is independent of lexical meaning. Neuroimage 14, 546-555.

January, D., Trueswell, J.C., Thompson-Schill, S.L., 2009. Co-localization of Stroop and syntactic ambiguity resolution in Broca's area: implications for the neural basis of sentence processing. J. Cogn. Neurosci. 21, 2434-2444.

Kaan, E., Harris, A., Gibson, E., Holcomb, P., 2000. The P600 as an index of syntactic integration difficulty. Lang. Cogn. Process. 15, 159-201.

Kang, A.M., Constable, R.T., Gore, J.C., Avrutin, S., 1999. An event-related fMRI study of implicit phrase-level syntactic and semantic processing. Neuroimage 10, 555-561.

Kaufman, R.C., Obler, L.K., 1995. Classification of normal reading error types. In: Leong, C.K., Joshi, R.M. (Eds.), Developmental and Acquired Dyslexia: Neuropsychological and Neurolinguistic Perspectives. Kluwer Academic Publishers, Dordrecht, pp. 149-157.

Kim, A., Osterhout, L., 2005. The independence of combinatory semantic processing: evidence from event-related potentials. J. Mem. Lang. 52, 205-225.

Kolk, H.H.J., Chwilla, D.J., 2007. Late positivities in unusual situations. Brain Lang. 100 257-261.

Kolk, H.H.J., Chwilla, D.J., Van Herten, M., Oor, P.J.W., 2003. Structure and limited capacity in verbal working memory: a study with event-related potentials. Brain Lang. 85, 1-36.

Kuperberg, G.R., 2007. Neural mechanisms of language comprehension: challenges to syntax. Brain Res. 1146, 23-49.

Kuperberg, G.R., McGuire, P.K., Bullmore, E.T., Brammer, M.J., Rabe-Hesketh, S., Wright, I.C., Lythgoe, D.J., Williams, S.C.R., David, A.S., 2000. Common and distinct neural substrates for pragmatic, semantic, and syntactic processing of spoken sentences: an fMRI study. J. Cogn. Neurosci. 12, 321-341.

Kuperberg, G.R., Holcomb, P.J., Sitnikova, T., Greve, D., Dale, A.M., Caplan, D., 2003a. Distinct patterns of neural modulation during the processing of conceptual and syntactic anomalies. J. Cogn. Neurosci. 15, 272-293.

Kuperberg, G.R., Sitnikova, T., Caplan, D., Holcomb, P.J., 2003b. Electrophysiological distinctions in processing conceptual relationships within simple sentences. Cogn. Brain Res. 17, 117-129.

Kuperberg, G.R., Caplan, D., Sitnikova, T., Eddy, M., Holcomb, P., 2006. Neural correlates of processing syntactic, semantic, and thematic relationships in sentences. Lang. Cogn. Process. 21, 489-530. 
Kuperberg, G.R., Kreher, D.A., Sitnikova, T., Caplan, D.N., Holcomb, P.J., 2007. The role of animacy and thematic relationships in processing active English sentences: evidence from event-related potentials. Brain Lang. 100, 223-237.

Kuperberg, G.R., Sitnikova, T., Lakshmanan, B.M., 2008. Neuroanatomical distinctions within the semantic system during sentence comprehension: evidence from functional magnetic resonance imaging. Neuroimage 40, 367-388.

Kutas, M., Hillyard, S.A., 1984. Brain potentials during reading reflect word expectancy and semantic association. Nature 307, 161-163.

Kutas, M., Van Petten, C., Kluender, R., 2006. Psycholinguistics electrified II: 1994-2005 In: Traxler, M., Gernsbacher, M.A. (Eds.), Handbook of Psycholinguistics, 2nd ed. Elsevier, New York, pp. 659-724.

Levelt, W.J.M., 1983. Monitoring and self-repair in speech. Cognition 14, 41-104.

Mason, R.A., Just, M.A., Keller, T.A., Carpenter, P.A., 2003. Ambiguity in the brain: what brain imaging reveals about the processing of syntactically ambiguous sentences. J. Exp. Psychol. Learn. Mem. Cogn. 29, 1319-1338.

McCarthy, G., Wood, C.C., 1985. Scalp distributions of event-related potentials: an ambiguity associated with analysis of variance models. Electroencephalogr. Clin. Neurophysiol. 62, 203-208.

Mechelli, A., Gorno-Tempini, M.L., Price, C.J., 2003. Neuroimaging studies of word and pseudoword reading: consistencies, inconsistencies, and limitations. J. Cogn. Neurosci. 15, 260-271.

Meyer, M., Friederici, A.D., von Cramon, D.Y., 2000. Neurocognition of auditory sentence comprehension: event related fMRI reveals sensitivity to syntactic violations and task demands. Cogn. Brain Res. 9, 19-33.

Miller, E.K., Cohen, J.D., 2001. An integrative theory of prefrontal cortex function. Annu. Rev. Neurosci. 24, 167-202.

Moro, A., Tettamanti, M., Perani, D., Donati, C., Cappa, S.F., Fazio, F., 2001. Syntax and the brain: disentangling grammar by selective anomalies. Neuroimage 13, 110-118.

Münte, T.F., Heinze, H.-J., Matzke, M., Wieringa, B.M., Johannes, S., 1998. Brain potentials and syntactic violations revisited: no evidence for specificity of the syntactic positive shift. Neuropsychologia 36, 217-226.

Nee, D.E., Wager, T.D., Jonides, J., 2007. Interference resolution: insights from a metaanalysis of neuroimaging tasks. Cogn. Affect. Behav. Neurosci. 7, 1-17.

Newman, R.L., Connolly, J.F., 2004. Determining the role of phonology in silent reading using event-related brain potentials. Cogn. Brain Res. 21, 94-105.

Nichelli, P., Grafman, J., Pietrini, P., Clark, K., Lee, K.Y., Miletich, R., 1995. Where the brain appreciates the moral of a story. NeuroReport 6, 2309-2313.

Novick, J.M., Trueswell, J.C., Thompson-Schill, S.L., 2005. Cognitive control and parsing: reexamining the role of Broca's area in sentence comprehension. Cogn. Affect. Behav. Neurosci. 5, 263-281.

Osterhout, L., Holcomb, P.J., 1992. Event-related brain potentials elicited by syntactic anomaly. J. Mem. Lang. 31, 785-806.

Rockstroh, B., Müller, M., Cohen, R., Elbet, T., 1992. Probing the functional brain state during P300-evocation. 1992. J. Psychophysiol. 6, 175-184.

Rockstroh, B., Muller, M., Heinz, A., Wagner, M., Berg, P., Elbert, T., 1996. Modulation of auditory responses during oddball tasks. Biol. Psychol. 43, 41-55.
Stroop, J.R., 1935. Studies on interference in serial verbal reactions. J. Exp. Psychol. 18, 643-662.

Thompson-Schill, S.L., D'Esposito, M., Aguirre, G.K., Farah, M.J., 1997. Role of left inferior prefrontal cortex in retrieval of semantic knowledge: a reevaluation. Proc. Natl Acad. Sci. USA 94, 14792-14797.

Thompson-Schill, S.L., Bedny, M., Goldberg, R.F., 2005. The frontal lobes and the regulation of mental activity. Curr. Opin. Neurobiol. 15, 219-224.

Urbach, T.P., Kutas, M., 2002. The intractability of scaling scalp distributions to infer neuroelectric sources. Psychophysiology 39, 791-808.

Urbach, T.P., Kutas, M., 2006. Interpreting event-related brain potential (ERP) distributions: implications of baseline potentials and variability with application to amplitude normalization by vector scaling. Biol. Psychol. 72, 333-343.

Van de Meerendonk, N., Kolk, H.H.J., Chwilla, D.J., Vissers, C.T.W.M., 2009. Monitoring in language perception. Lang. Linguist. Compass 3, 1211-1224.

Van de Meerendonk, N., Kolk, H.H.J. Vissers, C.T.W.M. Chwilla, D.J. 2010. Monitoring in language perception: Mild and strong conflicts elicit different ERP patterns. J. Cogn. Neurosci. 22, 67-82.

Van Herten, M., Kolk, H.H.J., Chwilla, D.J., 2005. An ERP study of P600 effects elicited by semantic anomalies. Cogn. Brain Res. 22, 241-255.

Van Herten, M., Chwilla, D.J., Kolk, H.H.J., 2006. When heuristics clash with parsing routines: ERP evidence for conflict monitoring in sentence perception. J. Cogn. Neurosci. 18, 1181-1197.

Vasey, M.W., Thayer, J.F., 1987. The continuing problem of false positives in repeated measures ANOVA in psychophysiology: a multivariate solution. Psychophysiology 24, 479-486.

Vissers, C.T.W.M., Chwilla, D.J., Kolk, H.H.J., 2006. Monitoring in language perception: the effect of misspellings of words in highly constrained sentences. Brain Res. 1106, 150-163.

Vissers, C.T.W.M., Kolk, H.H.J., Van de Meerendonk, N., Chwilla, D.J., 2008. Monitoring in language perception: evidence from ERPs in a picture-sentence matching task. Neuropsychologia 46, 967-982.

Vosse, T., Kempen, G., 2000. Syntactic structure assembly in human parsing: a computational model based on competitive inhibition and a lexicalist grammar. Cognition 75, 105-143.

Wartenburger, I., Heekeren, H.R., Burchert, F., Heinemann, S., De Bleser, R., Villringer, A., 2004. Neural correlates of syntactic transformations. Hum. Brain Mapp. 22, 72-81.

Wilding, E.L., 2006. The practice of rescaling scalp-recorded event-related potentials. Biol. Psychol. 72, 325-332.

Ye, Z., Zhou, X., 2008. Involvement of cognitive control in sentence comprehension: evidence from ERPs. Brain Res. 1203, 103-115.

Ye, Z., Zhou, X., 2009a. Conflict control during sentence comprehension: fMRI evidence. Neuroimage 48, 280-290.

Ye, Z., Zhou, X., 2009b. Executive control in language processing. Neurosci. Biobehav. Rev. 33, 1168-1177.

Yeung, N., Botvinick, M.M., Cohen, J.D., 2004. The neural basis of error detection: conflict monitoring and the error-related negativity. Psychol. Rev. 111, 931-959. 